\begin{tabular}{|c|c|}
\hline Title & A utomatic Phase Compensation for Extremely Short Optical-Pulse Generation U sing W avelet Transform \\
\hline Author(s) & Hazu, K.; Narita, K.; Sekikawa, T.; Y amashita, M. \\
\hline Citation & $\begin{array}{l}\text { IEEE Journal of Quantum Electronics, 43(12), 1218-1226 } \\
\text { https://doi.org/10.1109/JQE.2007.907546 }\end{array}$ \\
\hline Issue Date & 2007-11 \\
\hline Doc URL & http:/hdl.handle.net/2115/32323 \\
\hline Rights & $\begin{array}{l}\text { C2007 IEEE. Personal use of this material is permitted. However, permission to reprint/republish this material for } \\
\text { advertising or promotional purposes or for creating new collective works for resale or redistribution to servers or lists, } \\
\text { or to reuse any copyrighted component of this work in other works must be obtained from the IEEE. IEEE, IEEE } \\
\text { Journal of Quantum Electronics, } 43(12), 2007, \text { p1218-1226 }\end{array}$ \\
\hline Type & article \\
\hline File Information & 04384663.pdf \\
\hline
\end{tabular}

Instructions for use 


\title{
Automatic Phase Compensation for Extremely Short Optical-Pulse Generation Using Wavelet Transform
}

\author{
K. Hazu, K. Narita, T. Sekikawa, and M. Yamashita
}

\begin{abstract}
We introduce the wavelet transform (WT) method to automatic phase compensation for the generation of extremely short optical pulses with a duration of a few to several femtoseconds. This method is based on the immediate negative feedback of the spectral phase which is directly reconstructed by the wavelet-transform analysis with no uncertainty in phase retrieval without any manual decision, unlike the Fourier transform method. It is shown that the wavelet-transform method is useful for automatic compensations for three types of ultrabroadband pulses with different characteristics, such as: 1) a strong chirp; 2) a 600-rad spectral-phase variation over one octave bandwidth; and 3) a complicated spectral phase as well as a structured intensity spectrum.
\end{abstract}

Index Terms-Automatic phase compensation, few- to mono-cycle optical pulse, over-octave bandwidth pulses, wavelet transform (WT).

\section{INTRODUCTION}

$\mathbf{R}$ ECENTLY, compression to a 2.8 -fs single pulse in the mono-optical-cycle region has been demonstrated [1] using a technique based on the negative feedback (FB) of the reconstructed spectral phase [2] for the strongly chirped pulse from a gas-filled hollow fiber. Moreover, it has been shown that the technique is useful for pulse compression using a photonic crystal fiber (PCF) [3], [4] and for further pulse compression (e.g., $2.6 \mathrm{fs}, 1.3$ cycles) based on induced phase modulation [5]. This phase-compensation technique with a bandwidth exceeding the octave consists of the combination of a phase manipulator with a spatial light modulator (SLM) [6], a phase characterizer using a modified spectral phase interferometry for direct electric-field reconstruction (M-SPIDER) [7], and computers for phase analysis and SLM control. However, the technique has one problem that FB phase compensation does not operate automatically. This fact prevents the technique from being widely utilized by those who are not familiar with ultrafast optics. This is mainly due to the fact that a decision by an observer is required in the process of the Fourier-transform (FT) analysis for the spectral phase reconstruction from the M-SPIDER signal when the spectral phase of ultrabroadband pulses is complicated and/or the intensity spectrum has a structure (see Section II).

On the other hand, in 2004, it was demonstrated that a novel analysis for extracting the spectral phase from the SPIDER signal, based on wavelet transform (WT), provides an alter-

Manuscript received March 26, 2007; revised August 1, 2007.

The authors are with the Department of Applied Physics, Hokkaido University and Japan Science and Technology Agency (JST), CREST, Sapporo 060-8628, Japan (e-mail: mikio@eng.hokudai.ac.jp).

Color versions of one or more of the figures in this paper are available online at http://ieeexplore.ieee.org.

Digital Object Identifier 10.1109/JQE.2007.907546 native method for phase retrieval [8], [9]. The purpose of this paper is to show that the use of the WT analysis (instead of the FT analysis) enables us to compensate for the phase automatically in the FB mode without any manual decision, even for pulses with an ultrabroadband, a complicated spectral phase, and/or a structured intensity spectrum.

\section{Problem of FT ANalysis AND ApPlication of WT}

In general, the M-SPIDER signal $D(\omega)$ is described by the following equation [7], [10], [11]:

$$
\begin{aligned}
D(\omega)= & I(\omega)+I(\omega-\Omega) \\
+ & \sqrt{I(\omega)} \sqrt{I(\omega-\Omega)} \exp [i\{\phi(\omega)-\phi(\omega-\Omega)\}] \\
& \times \exp \left(i \omega \tau_{d}\right) \\
+ & \sqrt{I(\omega)} \sqrt{I(\omega-\Omega)} \exp [-i\{\phi(\omega)-\phi(\omega-\Omega)\}] \\
& \times \exp \left(-i \omega \tau_{d}\right)
\end{aligned}
$$

where $\Omega$ is the spectral shear, $\tau_{d}$ is the delay time between two replica pulses, $I(\omega)$ is the intensity spectrum, and $\phi(\omega)$ is the spectral phase of the pulse to be characterized. The first and second terms of (1) represent the dc component, the third term represents the + ac component including the spectral phase information, and the fourth term represents the -ac component. The spectral phase $\phi(\omega)$ is usually reconstructed from the measured M-SPIDER signal using the FT analysis through the following processes.

Step 1) Calculate the inverse FT of the M-SPIDER signal.

Step 2) Determine the lower $\left(T_{1}\right)$ and upper $\left(T_{2}\right)$ time limits of the eighth-order super-Gaussian filtering function to extract the +ac component (see Fig. 1.

Step 3) Calculate the FT of only the +ac component and its argument $\theta^{\prime}(\omega)=\phi(\omega)-\phi(\omega-\Omega)+\omega \tau_{d}$.

Step 4) Remove the linear term $\omega \tau_{d}$ from the $\theta^{\prime}(\omega)$ and integrate the spectral phase difference $\theta(\omega)=\phi(\omega)-$ $\phi(\omega-\Omega)$, that is, the $\theta(\omega)$ is an approximation for the first derivative of $\phi(\omega)$, multiplied with $\Omega$.

Fig. 1 is one example of results after the step 1), which was calculated from the M-SPIDER signal measured for the PCF output [3], [4]. Fig. 1(a) indicates that it is difficult to determine manually suitable values $T_{1}$ and $T_{2}$ quickly, even with the help of the comparison with the calculated result of the inverse FT of the corresponding replica spectrum. Different values $T_{1}$ and $T_{2}$ lead to different spectral phases [see Fig. 1(b)] and, hence, different temporal intensity profiles. In (1), in general, it is possible to separate dc and + ac components by choosing large $\tau_{d}$ for an optical pulse whose intensity spectrum is clear and not ultrabroad [13]. However, it becomes difficult to separate those components when the optical pulse spectrum has a bandwidth as ultrabroad as that of nearly one octave and is complicated because 

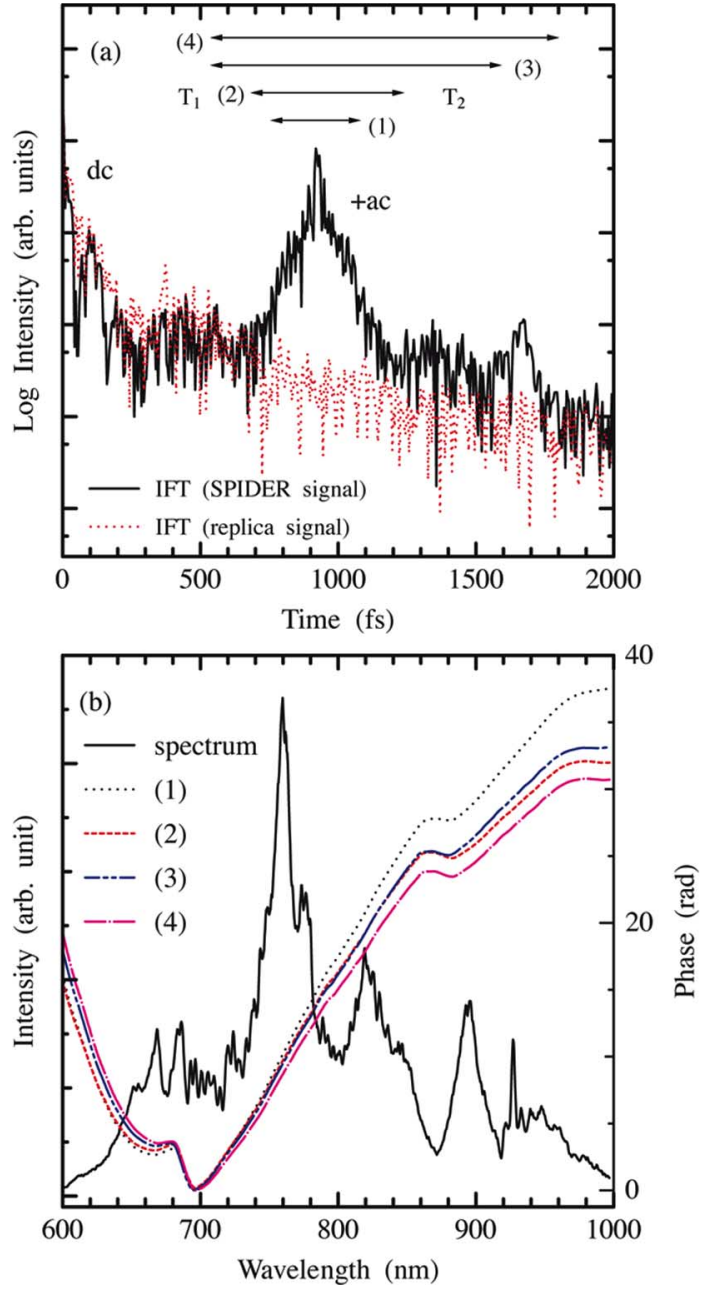

Fig. 1. (a) Result of calculating the inverse FT of the M-SPIDER signal (solid curve) and that of one of its replica spectra (dotted curve) which were measured for the PCF output [3], [4]. It is difficult to set apart dc and +ac components. Arrows indicate the lower $\left(T_{1}\right)$ and upper $\left(T_{2}\right)$ time limits of an eighth-order super-Gaussian filtering function to extract the + ac component. (1) $T_{1}=760$ to $T_{2}=1080 \mathrm{fs}$. (2) $T_{1}=680$ to $T_{2}=1240 \mathrm{fs}$. (3) $T_{1}=530$ to $T_{2}=1600$ fs. (4) $T_{1}=530$ to $T_{2}=1800 \mathrm{fs}$. (b) The intensity spectrum (solid curve) and reconstructed spectral phases using different time limits $\left(T_{1}\right.$ and $\left.T_{2}\right)$ of an eighth-order super-Gaussian filtering function in (a).

the + ac component extends so as to overlap in the time region after the inverse FT. Moreover, large $\tau_{d}$ results in a SPIDER signal with a decent number of interference fringes and hence request a measurement with a high spectral resolution for its signal [13]. For example, for large $\tau_{d}=1 \mathrm{ps}$, the spectral resolution necessary for a spectrometer is evaluated to be $0.05 \mathrm{~nm}$ at $400 \mathrm{~nm}$ by considering the fringe period of about $\tau_{d}$ and the Nyquist sampling criterion. This is the almost limited value, in practice, for the measurement of the present ultrabroad SPIDER signal.

To overcome this problem, we introduce the WT to the spectral phase analysis from the M-SPIDER signal in the FB phasecompensation technique. In addition, the WT analysis has additional advantages of fewer reconstruction processes, no uncertainty in phase retrieval, and usually higher accuracy [9] compared with the FT analysis. The WT converts the one-dimensional (1-D) frequency $(\omega)$ signal, the M-SPIDER signal $D(\omega)$, into the two-dimensional (2-D) time $(2 \pi / a)$-frequency $(b)$ topography $W(a, b)$. Using the Gabor wavelet $\psi(x)$, the complex WT equation $W(a, b)$ is expressed as follows [8]:

$$
\begin{aligned}
W(a, b) & =\int_{-\infty}^{\infty} \frac{1}{a} D(\omega) \psi\left(\frac{\omega-b}{a}\right) d \omega \\
\psi(x) & =\frac{1}{\sqrt{\pi} \sigma} \exp \left(-\frac{x^{2}}{\sigma^{2}}\right) \exp (-i x)
\end{aligned}
$$

where $a(\mathrm{rad} \cdot \mathrm{Hz})$ is the dilation factor, $b(\mathrm{rad} \cdot \mathrm{Hz})$ is the moving factor, and $\sigma$ is the window factor. In the computer calculation of $W(a, b)$, it is discretely computed at equal intervals for the parameters, where the mesh numbers of $a, b$, and $\omega$ are 543, $3072-7168$, and 3072-7168, respectively, depending on the broadening of the pulse intensity spectrum. As the FT has no time parameter, the chosen time window (width $T_{1}-T_{2}$ of the super-Gaussian filter after the FT) affects the signal (after the inverse transform) globally, complicating an automated optimization. In contrast, the WT does have a time parameter $(2 \pi / a)$, whose variation affects the transformed signal only locally (at the chosen "moving factor" $b$ ). Hence, the simple maximum-modulus (of $|W(a, b)|$ ) criterion can be applied for an automated optimization and determination of the phase trace. The spectral phase reconstruction using the WT is carried out through the following processes.

Step 1) Calculate the 2-D mapping $|W(a, b)|$, the absolute of (2) [see Fig. 2(c)], and find $a=a_{\max }$ where $|W(a, b)|$ at each value of $b$ becomes maximum.

Step 2) Calculate the argument of $W\left(a_{\max }, b\right)$, which corresponds to the above-mentioned $\theta^{\prime}(\omega)$, and integrate the spectral phase difference $\theta(\omega)$ after subtraction of the $\omega \tau_{d}$ as in steps 3) and 4) in the FT analysis. Those processes do not require any manual decisions.

To choose a suitable $\sigma$ value, the WT simulation analysis was carried out in the wide range of $\sigma=3 \sim 30$ for different numerically-given signals of SPIDER including the cases I, A and B (see Sections III, IV and V). As a result, the value of $\sigma=20$ was employed in this paper.

\section{Demonstration of Automatic Phase Compensation}

The experimental setup was similar to our previously reported one [3], [4] except for the two following points. First, an original computer program was added for the phase analysis by the WT method enabling the reconstruction automatically. Second, a 7.5-mm-long glass (BK7) as a dispersion medium yielding a strong chirp for 12-fs input pulses was employed.

A mode-locked Ti:sapphire laser was used as a light source. The laser producing a pulse duration of $12 \mathrm{fs}$ at a repetition rate of $75 \mathrm{MHz}$ was tuned to a 786-nm center wavelength $\left(\lambda_{0}=\right.$ $\left.2 \pi c / \omega_{0}\right)$ with a $620-\mathrm{mW}$ average power. The laser spectrum was broadened from 680 to $910 \mathrm{~nm}$ (see Fig. 2(a): the spectrum after passing through a 4-f phase compensator). The laser output pulse was divided into two beams with a 1:3 beam splitter. The lower intensity pulse was sent to the M-SPIDER apparatus as a reference chirped pulse. The higher intensity pulse was passed through the BK7 dispersion glass. After that, the strongly chirped pulse was directed into a 4 -f phase compensator. The 4-f system consisted of a pair of 500 grooves $/ \mathrm{mm}$ gratings, a pair 

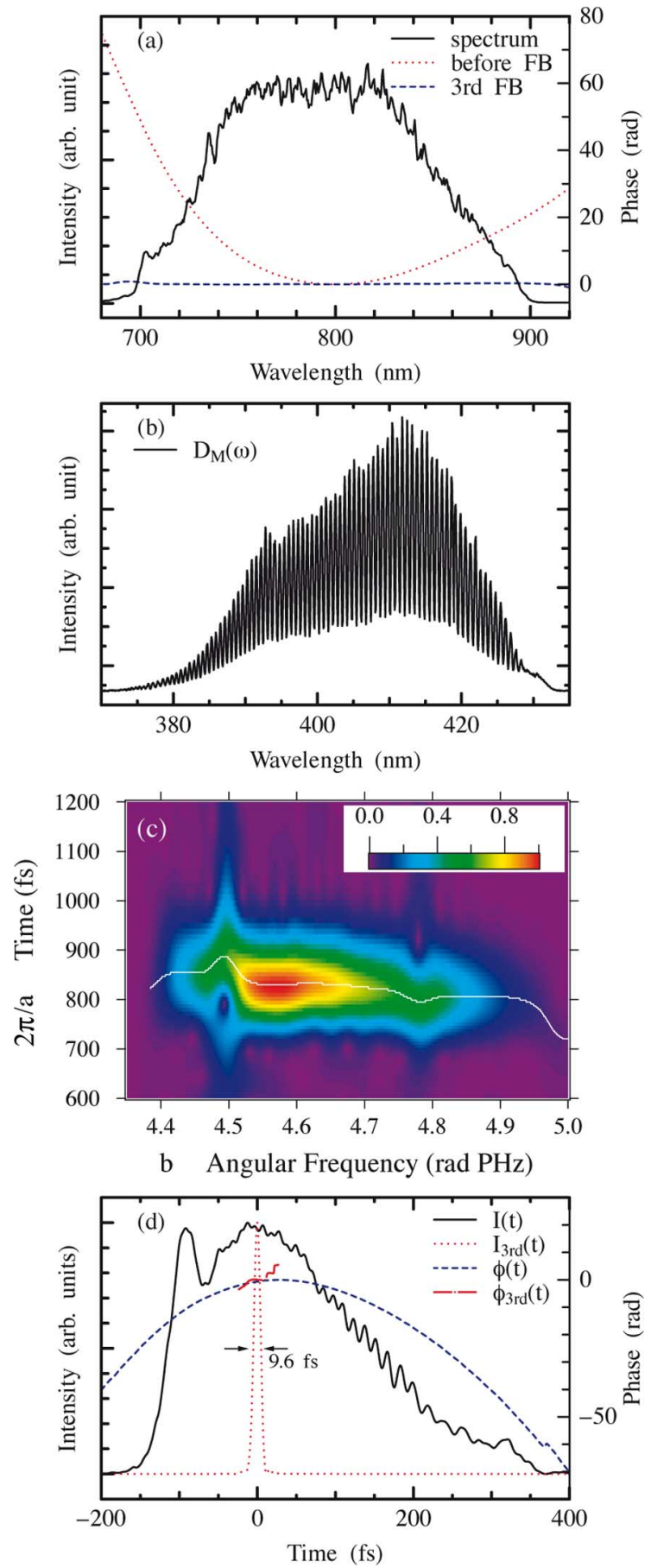

Fig. 2. Experimental result of automatic phase compensation for pulses which propagated through a BK7 glass with a 7.5-mm length. (a) The solid curve is the intensity spectrum. Dotted and dashed curves are reconstructed spectral phase before and after the third FB compensations, respectively. (b) M-SPIDER signal before FB compensation. (c) Two-dimensional map of $|W(a, b)|$ and the corresponding maximum-trace curve $\left|W\left(a_{\max }, b\right)\right|$. (d) Solid and dotted curves are reconstructed temporal intensity profiles before $(I(t))$ and after the third $\left(I_{3 \mathrm{rd}}(t)\right)$ FB compensations, respectively. Dashed and dashed-dotted curves are temporal phase profiles before $(\phi(t))$ and after the third $\left(\phi_{3 \mathrm{rd}}(t)\right)$ FB compensations, respectively. The pulse widths are 270 and $9.6 \mathrm{fs}$ for before and after third FB compensations, respectively.

of silver-coated mirrors, a pair of silver-coated concave mirrors with a 200-mm focal length, and an SLM with a 648 pixels, a $97-\mu \mathrm{m}$ pixel gap, and an $85 \%$ transmission at $800 \mathrm{~nm}$.
The output pulse from the 4-f phase compensator was guided to the M-SPIDER apparatus for characterization. The pulse was split into two beams with a 1:4 beam splitter, and a delay time $\tau_{d}$ between their pulses was controlled by a Michelson interferometer arm. The $\tau_{d}=868 \mathrm{fs}$ was determined from the measurement of the second-harmonic interference between pulses which were focused onto the type-I $\beta$-barium borate (BBO) crystal $(25-\mu \mathrm{m}$ thickness) by a $50-\mathrm{mm}$-focal-length parabolic mirror. The reference chirp pulse was produced by transmission through TF5 glass with a 10-cm length. Combined replica and reference pulses were superimposed on a surface of a type-II BBO crystal (50- $\mu \mathrm{m}$ thickness) by a $50-\mathrm{mm}$-focal-length parabolic mirror to produce two sum-frequency waves (those center angular frequencies $\omega_{\text {sum1 }}^{c}, \omega_{\text {sum2 } 2}^{c}$ ). The interference signal of their waves, the M-SPIDER signal, was detected by a combination of a 0.5 -m-focal-length monochromator with a 1200grooves/mm grating (Bruker 500 IS 2-0411) and an intensified charge-coupled device (ICCD: ANDOR DH 520-25F-03). The spectral resolution is $0.05 \mathrm{~nm}$ at $400 \mathrm{~nm}$. The spectral shear $\Omega(=53.3 \mathrm{rad} \cdot \mathrm{THz})$ and the spectral shift $\omega_{s}\left(=\omega_{\text {sum } 1}^{c}+\Omega / 2-\right.$ $\omega_{0}=2.29 \mathrm{rad} \cdot \mathrm{PHz}$ ) were measured from the comparison between spectra of two sum-frequency waves.

To generate the shortest pulse, we carried out the FB spectral phase compensations three times as well as the spectral phase measurement before FB. Fig. 2(a) shows the spectral phases before FB compensation (dotted curve) and after the third FB (dashed curve). All spectral phases before and after FB compensations were reconstructed automatically by the WT method $(\sigma=20)$. In addition, Fig. 2(c) shows the absolute WT function $|W(a, b)|$ and the corresponding maximum-trace curve $\left|W\left(a_{\max }, b\right)\right|$. Those were calculated from the measured M-SPIDER signal $D_{M}(\omega)$ before FB [Fig. 2(b)]. For one FB loop for phase compensation, it typically took about $81 \mathrm{~s}$ using the combination of two conventional personal computers, one for the M-SPIDER signal measurement and the other for the signal analysis and SLM control (this time is slightly shorter than that (at least $83 \mathrm{~s}$ ) in the case of the FT analysis), that is, it took $40 \mathrm{~s}$ to measure the M-SPIDER signal ranging from 370 to $435 \mathrm{~nm}$ owing to the requirement of three automatic rotations of the spectrometer grating for the present ultrabroadband pulse, under the condition of 5000 pulse accumulations per each grating angle. Then, it took $26 \mathrm{~s}$ for the WT analysis and $15 \mathrm{~s}$ to apply the negative spectral phase to the SLM and to control it. The former time (26 s) is comparable to the case of the FT analysis even with the use of the fast FT program (23 s) except for the manual decision time (at least $5 \mathrm{~s}$ ) because of the requirement of more steps (see the previous section). To reduce the FP loop time, the use of an upgraded spectrometer ICCD and computers, the program improvement, the parameter selection of the accumulation number [12], and the spectral shear and the delay time suitable for pulses to be characterized will all be useful. In particular, the use of the fast algorithms for the discrete WT with unequal grid spacing such as a so-called gdyadich grid for the $a$ and $b$ parameters [14] may shorten the computation time.

The chirped pulse before FB compensation has a spectral phase variation over $80 \mathrm{rad}$ in the spectral range from 680 to $910 \mathrm{~nm}$ and a temporal broadening from -200 to $+400 \mathrm{fs}$ with a duration of $270 \mathrm{fs}$ [Fig. 2(d)]. The fitted group delay dispersion (GDD) and third-order dispersion (TOD) are $+707 \mathrm{fs}^{2}$ and 
$+1462 \mathrm{fs}^{3}$ at $800 \mathrm{~nm}$, respectively. Those are consistent with the GDD and TOD of the dispersion glass and other optical components. The time-dependent phase [Fig. 2(d)] shows a strong chirp. After the third FB compensation, the spectral phase is almost flat with the smaller GDD than $10 \mathrm{fs}^{2}$ [Fig. 2(a)]. The corresponding temporal intensity profile [Fig. 2(d)] shows a duration of $9.6 \mathrm{fs}$, which is very close to the transform-limited (TL) pulse duration of $9.3 \mathrm{fs}$. These results demonstrate that the WT method is very useful for the automatic phase compensation even for pulses with a strong chirp and an ultrabroad spectrum.

\section{STUDY OF EXTREME PULSES}

\section{A. Case of Pulses With Over-Octave Bandwidth}

We investigated the possibility of the application of the WT analysis for automatic phase compensation of output pulses that were generated in our previous experiment of a gas-filled hollow fiber for the 2.8 -fs ( 1.5 cycles) pulse compression $\left[I_{c}(t)\right.$ in Fig. 3(e)] [1].

For this purpose, we performed two types of studies. The first is the simulation study for the test of the reliability of the WT analysis by comparison between a numerically given $\phi_{T}(\omega)$ (as "true" spectral phase) and the corresponding reconstructed $\phi_{R}^{T}(\omega)$ spectral phases for the over-octave bandwidth pulse, that is, the M-SPIDER signal $D_{T}(\omega)$ to be employed [Fig. 3(c)] was calculated from a numerically given intensity spectrum $I_{T}(\omega)$ (460 to $1060 \mathrm{~nm}$ ) [Fig. 3(a)] and a numerically given spectral phase $\phi_{T}(\omega)$ (over 600-rad variation) [Fig. 3(a)]. Their numerical data of $I_{T}(\omega)$ and $\phi_{T}(\omega)$ in this subsection $A$ are originally the ones which had been measured before FB compensation in the above-mentioned experiment [1] with parameters of $\Omega=26.7 \mathrm{rad} \cdot \mathrm{THz}$ and $\tau_{d}=930 \mathrm{fs}$. We chose the reconstruction of the most frequency-dependent spectral phase before FB because it is the most difficult automatic reconstruction work among those before and after FB compensations. From the $D_{T}(\omega)$, the spectral phase $\phi_{R}^{T}(\omega)$ was reconstructed by the WT method without any manual decision [Fig. 3(a)]. The reconstructed result $\phi_{R}^{T}(\omega)$ with the fitted GDD of $+476.3 \mathrm{fs}^{2}$ and TOD of $+250.5 \mathrm{fs}^{3}$ at $515 \mathrm{~nm}$ shows an excellent agreement with the given spectral phase $\phi_{T}(\omega)$ with the GDD of +480.2 $\mathrm{fs}^{2}$ and TOD of $+266.1 \mathrm{fs}^{3}$, over the whole frequency range exceeding the octave. This is also confirmed by the curve of the spectral phase difference $\phi_{R}^{T}(\omega)-\phi_{T}(\omega)$ as shown in Fig. 3(b). The analysis time ( $45 \mathrm{~s})$ was somewhat longer than the conventional FT analysis time ( $30 \mathrm{~s})$. This is because the data of the M-SPIDER signal obtained under six grating rotations, which were required for the over-octave bandwidth pulse, are much more than those of the corresponding signal in the previous section. The agreement between the reconstructed temporal intensity profile $I_{R}^{T}(t)$ and the given one $I_{T}(t)$ is also excellent over the whole time region from -520 to $+250 \mathrm{fs}$, as shown in Fig. 3(e).

Next, using the WT method, we studied the direct analysis from the M-SPIDER signal $D_{M}(\omega)(280$ to $460 \mathrm{~nm})$ measured before FB compensation [Fig. 3(d)] in the same 2.8-fs pulse experiment [1], where the fringe contrast of the signal $D_{M}(\omega)$ was much reduced compared with the $D_{T}(\omega)$. Fig. 3(b) shows the curve of the difference between the reconstructed $\phi_{R}^{M}(\omega)$ and given $\phi_{T}(\omega)$ spectral phases before FB. As a result, a similar
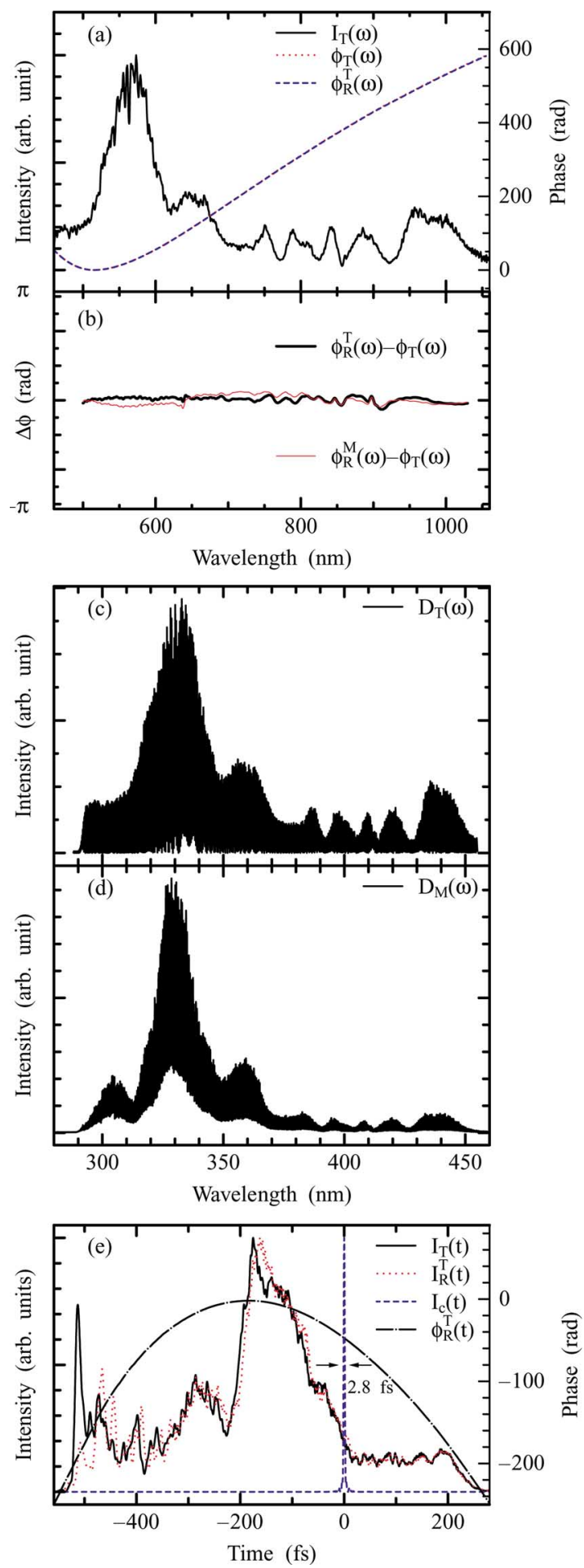

Fig. 3. Case A : Over-octave bandwidth pulses which propagated a gas-filled hollow fiber [1]. (a) The solid curve is the ultrabroadband spectrum $\left(I_{T}(\omega)\right)$. Dotted and dashed curves are the given spectral phase $\left(\phi_{T}(\omega)\right)$ and the reconstructed spectral phase $\left(\phi_{R}^{T}(\omega)\right.$ : see the text), respectively. (b) Solid and thin solid curves are the spectral phase difference of $\phi_{R}^{T}(\omega)-\phi_{T}(\omega)$ and that of $\phi_{R}^{M}(\omega)-\phi_{T}(\omega)$, respectively. ( $\phi_{R}^{M}(\omega)$ : see the text). (c) Calculated M-SPIDER signal $\left(D_{T}(\omega)\right)$. (d) Measured M-SPIDER signal $\left(D_{M}(\omega)\right)$. (e) Solid, dotted and dashed curves are given $\left(I_{T}(t)\right)$, reconstructed $\left(I_{R}^{T}(t)\right)$ and compressed $\left(I_{c}(t):[1]\right)$ temporal intensity profiles, respectively. The dashed-dotted curve is the temporal phase $\left(\phi_{R}^{T}(t)\right)$ before FB.

agreement to the result shown above was confirmed. This suggests that the WT analysis is useful for the automatic phase com- 
pensation of extreme pulses with a large spectral-phase variation of more than $600 \mathrm{rad}$ in the frequency region exceeding the octave.

Since the $D_{T}(\omega)$ is the numerically given signal of SPIDER, it has no noise and a clean fringe contrast. On the other hand, the $D_{M}(\omega)$ is the experimentally measured signal [1] and, hence, has usually noise which depends on the conditions of pulses to be characterized and the measurement instrument. As a result, the SPIDER signal $D_{M}(\omega)$ with a much reduced fringe contrast is observed. However, as shown in [13], the fringe contrast is fairly insensitive to the spectral phase reconstruction. This is because the SPIDER algorithm is not concerned with the amplitude of the interferogram. It is concerned only with the spacing of the interference fringe [13].

\section{B. Case of Pulses With Complicated Spectral Phase and Structured Intensity Spectrum}

Next, we investigated the possibility of the WT-analysis application for automatic phase compensation of output pulses generated from a PCF (zero-dispersion wavelength of $853 \mathrm{~nm}$ ) in our previous experiment of 5.8-fs pulse compression $\left[I_{c}(t)\right.$ in Fig. 4(e)] [4].

In the same manner as in Section IV-A, the M-SPIDER signal $D_{T}(\omega)$ to be employed [Fig. 4(c)] was calculated from the complicated spectral phase $\phi_{T}(\omega)$ before FB compensation (over 30-rad variation) [Fig. 4(a)] and the structured intensity spectrum $I_{T}(\omega)$ (600 to $1010 \mathrm{~nm}$ ) [Fig. 4(a)] which had been measured in [4] with parameters of $\Omega=57.6 \mathrm{rad} \cdot \mathrm{THz}$ and $\tau_{d}=922$ fs. From the $D_{T}(\omega)$, the complicated spectral phase $\phi_{R}^{T}(\omega)$ was reconstructed by the WT method with a good reproducibility but without any manual decision [Fig. 4(a)]. Although the spectral phase is so complicated that it is difficult to find a suitable polynomial function, the agreement between the reconstructed $\phi_{R}^{T}(\omega)$ and given $\phi_{T}(\omega)$ spectral phases is excellent over the whole frequency, except for the following point [Fig. 4(a) and (b)], that is, the small phase error occurred at wavelengths of $\sim 680 \mathrm{~nm}\left(B_{1}\right)$ and $\sim 860 \mathrm{~nm}\left(B_{2}\right)$ in Fig. 4(b). This disagreement will be discussed in the next section. The analysis time $(32 \mathrm{~s})$ was a little longer than the FT-analysis time (28 s). As for the temporal intensity profiles $I_{R}^{T}(t)$ and $I_{T}(t)$, the agreement is good over the whole time region from -100 to $+100 \mathrm{fs}$, including many subpulse profiles [Fig. 4(e)]. Furthermore, the direct WT analysis of the measured M-SPIDER signal $D_{M}(\omega)$ [Fig. 4(d)] [4] showed that the same conclusion as that of the above-presented simulation study is derived. This suggests that the WT method is useful for the atomatic phase compensation of extreme pulses with a structured intensity spectrum and a complicated spectral phase.

\section{DISCUSSION}

It is valuable to compare the accuracy between the spectral phase $\phi_{R}^{\mathrm{WT}}(\omega)$ reconstructed by the WT method and the spectral phase $\phi_{R}^{\mathrm{FT}}(\omega)$ reconstructed by the FT method. This was performed on the basis of the same M-SPIDER signal $D_{T}(\omega)$ calculated from the measured spectral phase $\phi_{T}(\omega)$ and intensity spectrum $I_{T}(\omega)$ with experimental parameters of $\Omega$ and $\tau_{d}$. That is, we introduced a root-mean-square (rms) spectral-phase error
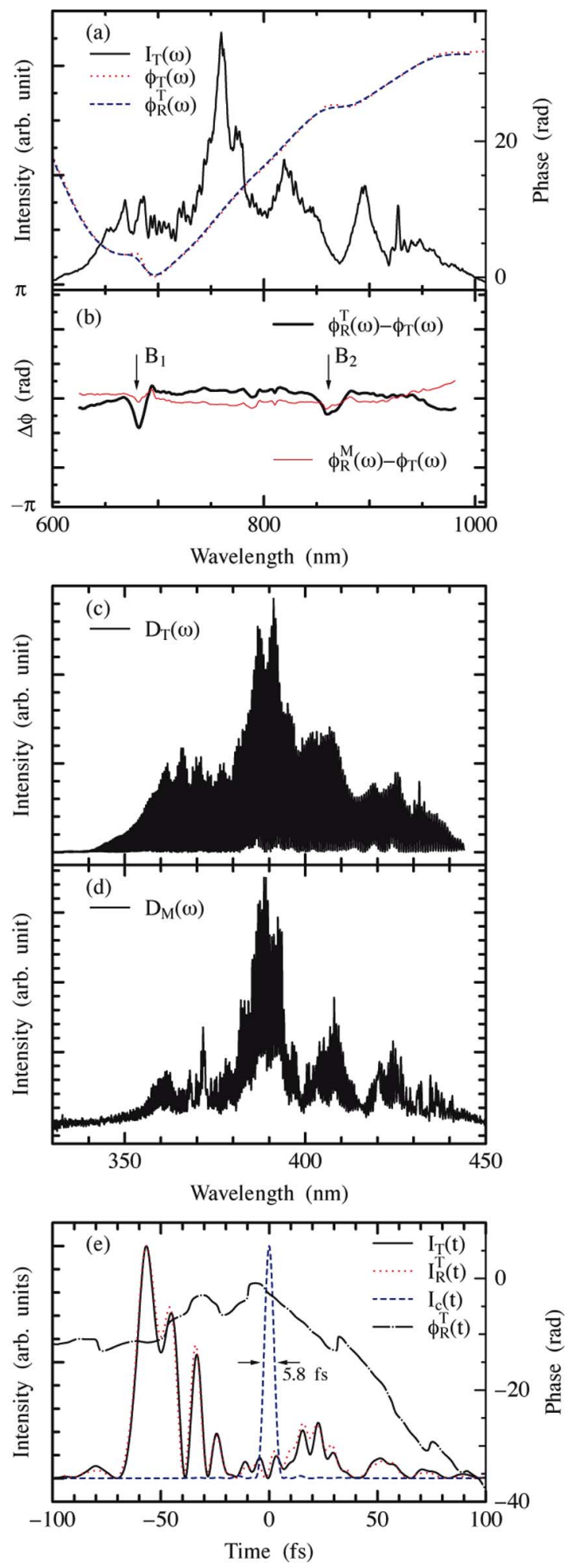

Fig. 4. Case B: complicated spectral-phase pulses with a structured spectrum, which propagated a PCF [4]. (a)-(e) correspond to Fig. 3(a)-(e), respectively.

$\varepsilon_{\phi}^{i}$ [13] for a quantitative comparison between the $\phi_{R}^{i}(\omega)(i=$ WT or FT) and the $\phi_{T}(\omega)$ as follows:

$\varepsilon_{\phi}^{i}=\frac{\sqrt{\frac{1}{N} \sum_{j=1}^{N}\left[\phi_{R}^{i}\left(\omega_{j}\right)-\phi_{T}\left(\omega_{j}\right)-C_{1} \omega_{j}-C_{2}\right]^{2} \cdot I_{T}^{2}\left(\omega_{j}\right)}}{\sqrt{\frac{1}{N} \sum_{j=1}^{N} I_{T}^{2}\left(\omega_{j}\right)}}$,
$i=\mathrm{WT}$ or FT $, j=1,2, \ldots, N$ 
TABLE I

RMS SPECTRAL-PHASE ERROR

\begin{tabular}{c|c|c}
\hline CASE & $\varepsilon_{\phi}^{W T}(\mathrm{rad})$ & $\varepsilon_{\phi}^{F T}(\mathrm{rad})$ \\
\hline $\mathrm{I}$ & $1.02 \times 10^{-3}$ & $6.85 \times 10^{-4}$ \\
\hline $\mathrm{A}$ & $5.00 \times 10^{-3}$ & $3.93 \times 10^{-3}$ \\
\hline $\mathrm{B}$ & $54.6 \times 10^{-3}$ & $25.8 \times 10^{-3}$ \\
\hline
\end{tabular}

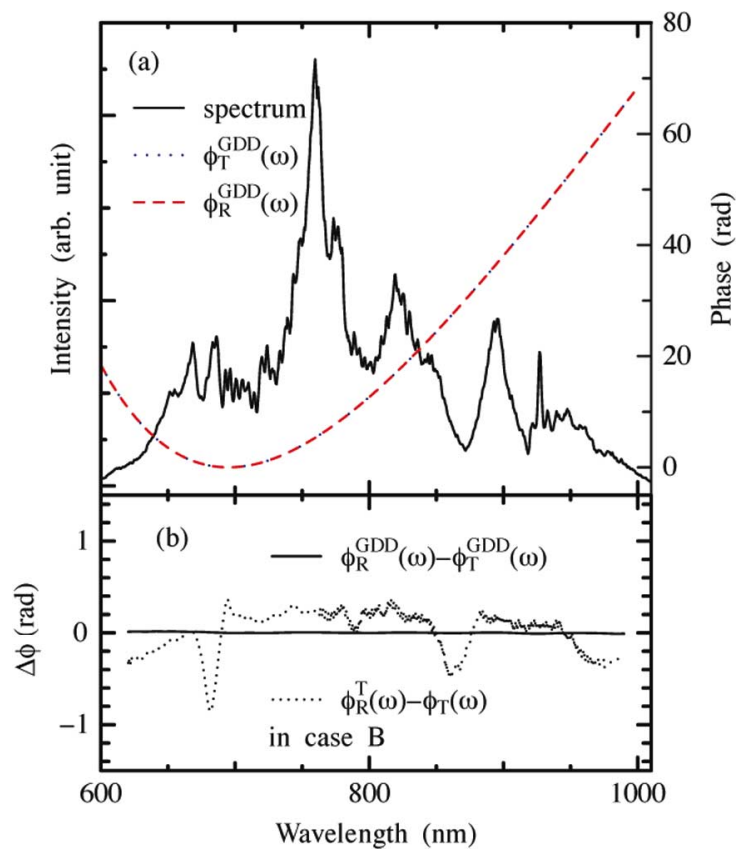

Fig. 5. (a) Intensity spectrum (solid curve) of case B and the spectral phase $\left(\phi_{T}^{G D D}(\omega)\right.$ : dotted curve), which were employed in the simulation to clarify the cause of the spectral phase error. The dashed curve is the corresponding reconstructed spectral phase $\left(\phi_{R}^{\mathrm{GDD}}(\omega)\right)$, which overlaps with the $\phi_{T}^{\mathrm{GDD}}$. (b) The spectral phase difference of $\phi_{R}^{\mathrm{GDD}}(\omega)-\phi_{T}^{\mathrm{GDD}}(\omega)$ (solid curve) and that of $\phi_{R}^{T}(\omega)-\phi_{T}(\omega)$ in case B (dotted curve).

where $C_{1}$ of the group delay term and $C_{2}$ of the constant phase term are given as constant values so that the phase error is minimized for both WT and FT methods, that is, attention is paid to the GDD and higher-order dispersion terms which are only meaningful for the SPIDER measurement. These values of $C_{1}$ and $C_{2}$ in the WT method are different from those in the FT method. For the FT method, the optimized time limits $T_{1}$ and $T_{2}$ were employed. We focus the spectral phase $\phi_{R}^{i}(\omega)$ before FB phase compensation for the three cases described in Sections III (which we call case I) and IV (cases A and B) because the evaluation for compensation of the most strongly chirped pulse before FB, among pulses to be compensated before and after FB operations, is the most important. The result under the same mesh number $N$ of 1024 in (4) is given in Table I. For all of the cases, the rms phase error in both methods is small, and the accuracy of the WT method and that of the FT method are comparable. The error values are reasonable in comparison with the reported result in [13], with the help of an equivalence principle (Parseval's theorem) between time and frequency. In both methods, the rms error becomes larger in case B with the structured spectrum and the complicated spectral phase.

To clarify the above-mentioned result in more detail, we investigated the cause of the phase error at specific wavelengths $B_{1}$ and $B_{2}$ in case B [see Fig. 4(a) and (b)]. There are three

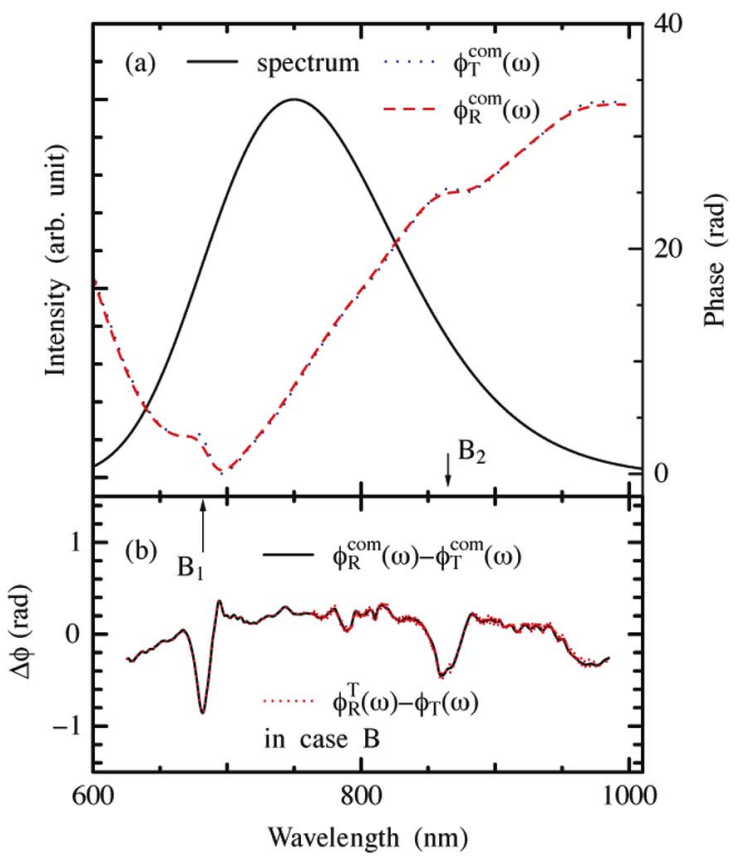

Fig. 6. (a) Gaussian intensity spectrum (solid curve) and the spectral phase $\left(\phi_{T}^{\text {com }}(\omega)\right.$ : dotted curve) of case B, which were employed in the simulation to clarify the cause of the spectral phase error. The dashed curve is the corresponding reconstructed spectral phase $\left(\phi_{R}^{\operatorname{com}}(\omega)\right)$, which almost overlaps with the $\phi_{T}^{\text {com }}(\omega)$ except for two wavelength regions $B_{1}$ and $B_{2}$. (b) Spectral phase difference of $\phi_{R}^{\operatorname{com}}(\omega)-\phi_{T}^{\operatorname{com}}(\omega)$ (solid curve) and that of $\phi_{R}^{T}(\omega)-\phi_{T}(\omega)$ in case B (dotted curve), which overlaps.

possibilities: the effect of the structure of the intensity spectrum, the effect of the complicated spectral phase such as step-like curves (e.g., discontinuous-like curves) and their combined effect. Fig. 5(a) shows the reconstructed spectral phase $\phi_{R}^{\mathrm{GDD}}(\omega)$ from the test SPIDER signal $D_{T}(\omega)$ which was calculated from the structured intensity spectrum $I_{T}(\omega)$ in case B and the given spectral phase $\phi_{T}^{\mathrm{GDD}}(\omega)$ with only the large GDD of $+200 \mathrm{fs}^{2}$ at $800 \mathrm{~nm}$. Fig. 5(b) shows the corresponding spectral-phase difference of $\phi_{R}^{\mathrm{GDD}}(\omega)-\phi_{T}^{\mathrm{GDD}}(\omega)$. The agreement between $\phi_{R}^{\mathrm{GDD}}(\omega)$ and $\phi_{T}^{\mathrm{GDD}}(\omega)$ is excellent. This fact indicates that the structured spectrum does not cause the phase error. In the same manner, we reconstructed the spectral phase $\phi_{R}^{\mathrm{com}}(\omega)$ from the test SPIDER signal $D_{T}(\omega)$ which was calculated from the spectral phase $\phi_{T}^{\operatorname{com}}(\omega)$ in case B and the Gaussian spectrum $I_{T}(\omega)$ ultrabroadened from 550 to $1050 \mathrm{~nm}$ (e.g., a bandwidth of $88.2 \mathrm{THz}$, a center wavelength of $750 \mathrm{~nm}$, and a TL pulse duration of $5.0 \mathrm{fs}$ ). Fig. 6(a) and (b) shows the reconstructed spectral phase $\phi_{R}^{\text {com }}(\omega)$ and the corresponding phase difference of $\phi_{R}^{\operatorname{com}}(\omega)-\phi_{T}^{\text {com }}(\omega)$, respectively. The result shows almost the same phase error as that in case $B$, in the same wavelength regions $B_{1}(\sim 680 \mathrm{~nm})$ and $B_{2}(\sim 860 \mathrm{~nm})$. Therefore, the phase error originates from the part of the step-like curve in the complicated spectral phase.

To get the quantitative knowledge concerning this point, we carried out the following simulation study, that is, the spectral phase $\phi_{R}(\omega)$ was reconstructed from the test SPIDER signal $D_{T}(\omega)$. It was calculated from the $0-\pi$ step-like phase curve with different slopes $(\pi / \Delta \lambda \mathrm{s})$ using a function of $\phi_{T}(\omega)=$ $\pi /\left\{1+\exp \left[2 \pi c\left(\omega^{-1}-\omega_{0}^{-1}\right) / a\right]\right\}$ with $a=(2 \pi c / 9.190) \times$ $\left(\Delta \omega /\left(\omega_{0}\right)^{2}\right)=\Delta \lambda / 9.190, \lambda_{0}=2 \pi c / \omega_{0}=800 \mathrm{~nm}$, and $\Delta \lambda=\left(2 \pi c / \omega_{0}^{2}\right) \Delta \omega=5 \sim 100 \mathrm{~nm}$ (Fig. 7(a) and Fig. 8) 

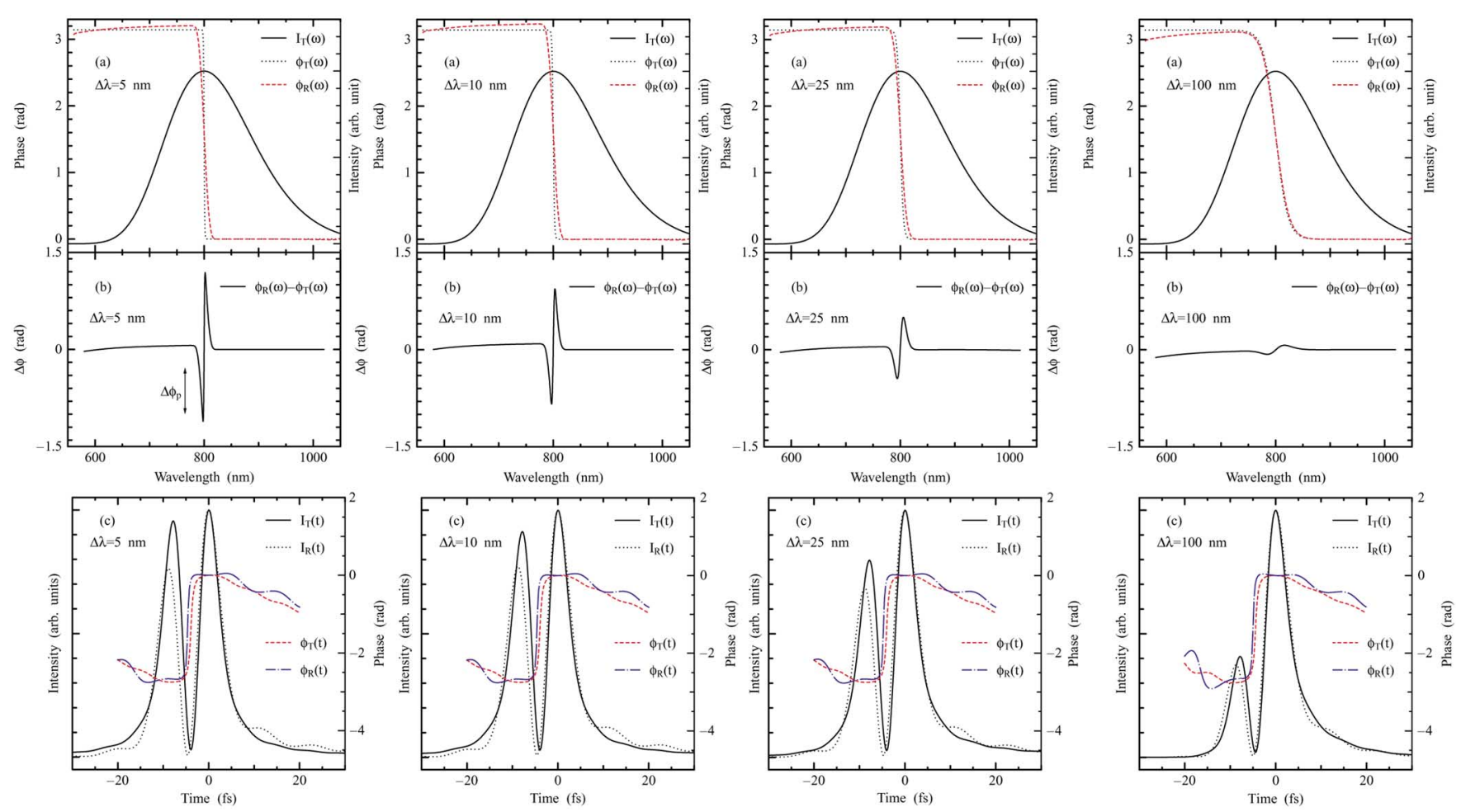

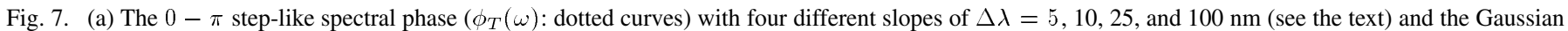

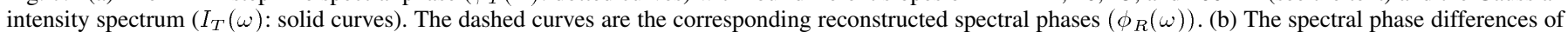

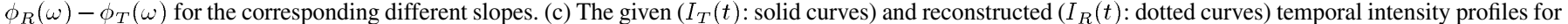
the corresponding different slopes. The dashed and dashed-dotted curves are the given $\left(\phi_{T}(t)\right)$ and reconstructed $\left(\phi_{R}(t)\right)$ time-dependent phases, respectively.

and the Gaussian spectrum with a bandwidth of $88.2 \mathrm{THz}$, a center wavelength of $800 \mathrm{~nm}$, and a TL pulse duration of $5.0 \mathrm{fs}$ [Fig. 7(a)]. The reconstructed spectral phase $\phi_{R}(\omega)$ and the corresponding phase difference $\Delta \phi(\omega)=\phi_{R}(\omega)-\phi_{T}(\omega)$ are shown in Fig. 7(a) and (b), respectively. The phase difference $\Delta \phi(\omega)$ shows the so-called dispersion-like curve. Its peak-to-zero difference $\Delta \phi_{p}$ [see Fig. 7(b) and Fig. 8] decreases rapidly in the range from the slope of $\pi / \Delta \lambda=\pi / 5$ to $\pi / 15$ $[\mathrm{rad} / \mathrm{nm}]$, and after the slope over $\pi / 50[\mathrm{rad} / \mathrm{nm}]$ the $\Delta \phi_{p}$ gradually approaches a difference of zero (Fig. 8). Fig. 7(c) shows the reconstructed temporal-intensity profile with a splitting and the reconstructed time-dependent phase. The decrease of the peak intensity difference $\Delta I_{s}=I_{s, T}-I_{s, R}$ (see Fig. 7(c) and Fig. 8) of the subpulse is considerably similar to the $\Delta \phi_{p}$ curve (Fig. 8), while the difference of the main pulse duration (5.1-5.7 fs) keeps almost constant. For example, the sharpness $(\pi / 10[\mathrm{rad} / \mathrm{nm}])$ of the spectral-phase slope difference around the wavelength $B_{1}$ in case $\mathrm{B}$ almost corresponds to that ( $\pi / 10$ $[\mathrm{rad} / \mathrm{nm}]$ ) in Fig. 7(a). Comparison between the corresponding maximum phase error ( $\left.\Delta \phi_{p}^{\mathrm{ex}} \cong 0.9 \mathrm{rad}\right)$ in case $\mathrm{B}$ and that $\left(\Delta \phi_{p}^{\text {si }} \cong 0.8 \mathrm{rad}\right)$ in Fig. 7(b) indicates a good agreement. A similar result was also confirmed for the comparison between the case of the wavelength $B_{2}$ and the case $(\pi / 25[\mathrm{rad} / \mathrm{nm}])$ of Fig. 7(a).

We also carried out the simulation for the effect of the step-like phase curve using the FT analysis. The result showed that the phase error in the range of the spectral-phase slope of $\pi / \Delta \lambda=\pi / 5$ to $\pi / 10[\mathrm{rad} / \mathrm{nm}]$ in the FT analysis is much larger than that in the WT analysis. Moreover, it is extremely

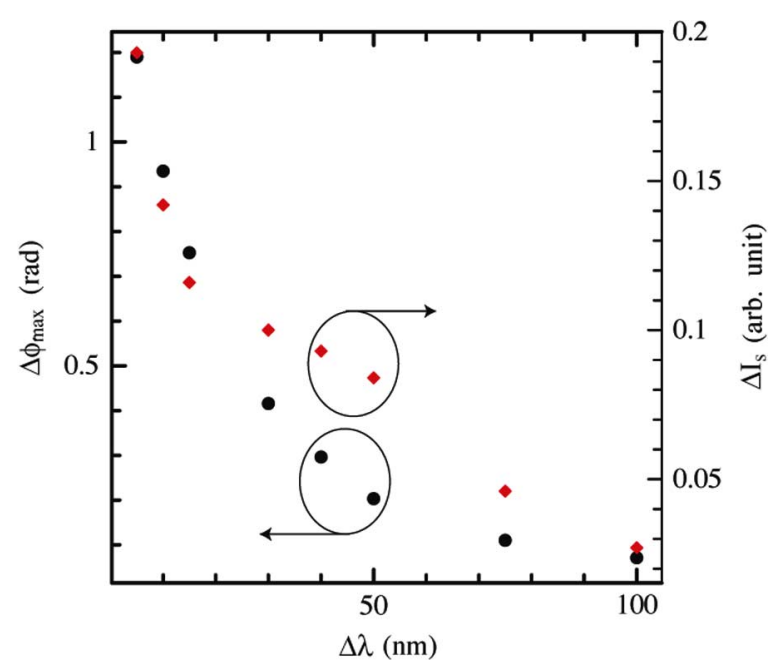

Fig. 8. Peak-to-zero difference $\left(\Delta \phi_{p}\right.$ : Solid circles) of the phase error curve [see $\phi_{R}(\omega)-\phi_{T}(\omega)$ in Fig. 7(b)] and the peak difference $\left(\Delta I_{s}\right.$ : Solid diamonds) between the subpulse of the $I_{T}(t)$ and that of the $I_{R}(t)$ [see Fig. 7(c)] as a function of the phase slope parameter $\Delta \lambda$ [see the text and Fig. 7(a)].

difficult to determine the suitable time limits of $T_{1}$ and $T_{2}$ for the filtering of the + ac component, that is, many trials (typically, more than five times) using variable $T_{1}$ and $T_{2}$ values were done, and it took a long time (typically several minutes) including judging time to find the robust spectral phase. It is much more difficult to do this work, compared with case I (in Section III) with such a nonstep-like spectral phase [the dotted 
curve in Fig. 2(a)]. Around the step-like phase change, the greater error in the FT method always yields more than that of the WT method even after many trials. On the other hand, the latter WT method is automatically carried out. As for this point, therefore, the WT analysis is superior to the FT analysis.

Finally, we would like to point out the influence of the error of the reconstructed phase on the FB phase compensation. The problem of the less-than-perfect reconstruction of the spectral phase, in the feedback technique, is not serious in practice. This is because we can repeat the FB compensation until complete phase compensation is achieved, and the small variation of the spectral phase after FB makes the correct WT analysis easier, even if the spectral phase was not reconstructed perfectly before FB. However, the FT method always requests suitable manual decisions (for $T_{1}$ and $T_{2}$ values) where the analyses using the pairs of their different values are carried out several times for the one SPIDER signal at least before FB operation. Under this condition, the traditional FT SPIDER can also achieve satisfactory results if the FB process is repeated for further several times. In contrast, the WT method enables us to reconstruct the suitable spectral phase automatically (without any manual decisions) at each FB and to achieve satisfactory results after a few FBs automatically.

\section{CONCLUSION}

We have demonstrated that the WT method for the analysis of the M-SPIDER signal is useful for automatic phase compensation of three different extreme pulses, that is, the use of the WT method has enabled us to compensate for a strong chirp of pulses with an ultrabroad spectrum automatically and to generate nearly-TL pulses of $9.6 \mathrm{fs}$ in duration. Furthermore, it has been shown, for over-octave bandwidth pulses with a large spectral-phase variation and ultrabroadband pulses with a complicated spectral phase as well as a structured spectrum, that the spectral phase reconstructed automatically by the wavelet transform method gives the correct spectral phase except for a few wavelength regions. We have revealed that the phase error occurring in these few regions is not due to the structure in the intensity spectrum but due to the step-like curve in the spectral phase. However, it has been pointed out that the problem of the phase error in the few regions is not serious in the repetitive FB compensation technique in practice to get complete phase compensation. We expect that this technique of the online phase control of extremely short optical pulses using the WT analysis will bring about a new point of view for fields such as selective chemistry, quantum control [15], nonlinear optical microscopy [2], and attosecond pulse control [16].

\section{REFERENCES}

[1] M. Yamashita, K. Yamane, and R. Morita, "Quasi-automatic phasecontrol technique for chirp compensation of pulses with over-one-octave bandwidth-Generation of few- to mono-cycle optical pulses," IEEE Sel. Top. Quantum. Electron., vol. 12, no. 2, pp. 213-222, Mar./ Apr. 2006.
[2] M. Yamashita, H. Shigekawa, and R. Morita, Mono-Cycle Photonics and Optical Scanning Tunneling Microscopy. Berlin, Germany: Springer-Verlag, 2005

[3] M. Adachi, K. Yamane, R. Morita, and M. Yamashita, "Pulse compression using direct feedback of the spectral phase from photonic crystal fiber output without the need for the Taylor expansion method," IEEE Photon. Technol. Lett., vol. 16, no. 8, pp. 1951-1953, Aug. 2004.

[4] M. Adachi, K. Yamane, R. Morita, and M. Yamashita, "Sub-5-fs pulse compression of laser output using photonic crystal fiber with short zero-dispersion wavelength," Jpn. J. Appl. Phys., vol. 44, no. 47, pp. L1423-L1425, 2005.

[5] E. Matsubara, K. Yamane, T. Sekikawa, and M. Yamashita, "Generation of 2.6-fs optical pulses using induced phase modulation in a gasfilled hollow fiber," J. Opt. Soc. Amer. B, vol. 24, no. 4, pp. 985-989, 2007.

[6] N. Karasawa, L. Li, A. Suguro, H. Shigekawa, R. Morita, and M. Yamashita, "Optical pulse compression to 5.0 fs by use of only a spatial light modulator for phase compensation," J. Opt. Soc. Amer. B, vol. 18 , no. 11 , pp. 1742-1746, 2001

[7] M. Hirasawa, N. Nakagawa, K. Yamamoto, R. Morita, R. Shigekawa, and M. Yamashita, "Sensitivity improvement of spectral phase interferometry for direct electric-field reconstruction for the characterization of low-intensity femtosecond pulses," Appl. Phys. B, vol. 74, pp. S225-S229, 2002.

[8] Y. Deng, Z. Wu, L. Chai, C. Wang, K. Yamane, R. Morita, M. Yamashita, and Z. Zhang, "Wavelet-transform analysis of spectral shearing interferometry for phase reconstruction of femtosecond optical pulses," Opt. Exp., vol. 13, no. 6, pp. 2120-2126, 2005.

[9] Y. Deng, C. Wang, L. Chai, and Z. Zhang, "Determination of Gabor wavelet shaping factor for accurate phase retrieval with wavelet-transform," Appl. Phys. B, vol. 81, no. 8, p. 1107, 2005.

[10] C. Iaconis and I. A. Walmsley, "Self-referencing spectral interferometry for measuring ultrashort optical pulses," IEEE J. Quantum Electron., vol. 35, no. 4, pp. 501-509, Apr. 1999.

[11] I. A. Walmsley, , F. X. Kärtner, Ed., "Characterization of ultrashort optical pulses in the few-cycle regime using spectral phase interferometry for direct electric-field reconstruction," in Few-Cycle Laser Pulse Generation and Its Applications. Berlin, Germany: Springer-Verlag, 2004, pp. 265-291.

[12] W. Kornelis, J. Biegert, J. W. G. Tisch, M. Nisoli, G. Sansone, C. Vozzi, S. De Silvestri, and U. Keller, "Single-shot kilohertz characterization of ultrashort pulses by spectral phase interferometry for direct electricfield reconstruction," Opt. Lett., vol. 28, no. 4, pp. 281-283, 2003.

[13] M. E. Anderson, L. L. L. de Araujo, E. M. Kosik, and I. A. Walmsley, "The effects of noise on ultrashort-optical-pulse measurement using SPIDER,” Appl. Phys. B, vol. 70, pp. S85-S93, 2000.

[14] O. Rioul and P. Duhamel, "Fast algorithms for discrete and continuous wavelet transforms," IEEE Trans. Inf. Theory, vol. 38, no. 2, pp. 569-586, Mar. 1992.

[15] M. Shapiro and P. Brumer, Principles of the Quantum Control of Molecular Processes. New York: Wiley, 2003.

[16] R. Bartels, S. Backus, E. Zeek, L. Misoguti, G. Vdovin, I. P. Christov, M. M. Murnane, and H. C. Kapteyn, "Shaped-pulse optimization of coherent emission of high-harmonic soft X-rays," Nature, vol. 406, no. 6792, pp. 164-166, 2000.

Kouji Hazu was born in Nagoya, Japan, in 1976. He received the Ph.D. degree in electrical, electronics, and computer engineering from Waseda University, Tokyo, Japan, in 2004.

In 2003, he joined the Department of Electrical, Electronics, and Computer Engineering, Waseda University, as a Research Associate. He studied biexcitons and exciton-exciton interaction in hexagonal semiconductors. In 2006, he joined the Department of Applied Physics, Hokkaido University, Sapporo, as a Postdoctoral Fellow (CREST). His current research interests are in mono-cycle optical pulse generation and its chirp compensation technique.

Dr. Hazu is a member of the Physical Society of Japan and the Japan Society of Applied Physics.

Keisuke Narita was born in Sapporo, Japan, in 1982. He received the B.E. degree from Hokkaido University, Sapporo, Japan, in 2005. He is currently working toward the M.S. degree at the Department of Applied Physics, Hokkaido University.

His current research interest is automatic chirp compensation of mono-cycle optical pulses using wavelet transform. 
Taro Sekikawa was born in Fujisawa, Japan, in 1967. He received the B.S., M.S., and Ph.D. degrees in physics from the University of Tokyo, Tokyo, Japan, in 1990,1992 , and 1996, respectively.

In 1996, he joined the Institute for Solid State Physics, University of Tokyo, where he was engaged in high harmonic generation and its application to solidstate physics. In 2005, he joined the Department Applied Physics, Hokkaido University, Sapporo, Japan, as an Associate Professor. His current research interests include attophysics and application of high harmonics to materials science.

Dr. Sekikawa is a member of the Optical Society of America and the Japan Society of Applied Physics.
Mikio Yamashita was born in Kyoto, Japan, on February 15, 1946. He received the Ph.D. degree from Kyoto University, Kyoto, Japan, in 1976, in electronics engineering.

From 1978 to 1979, he was a Visiting Research Associate with Imperial College, London, U.K. From 1970 to 1991, he was with the Electrotechnical Laboratory, Tokyo and Tsukuba, Japan. Since 1991, he has been with the Department of Applied Physics, Hokkaido University, Sapporo, Japan, as a Professor. He has published more than 100 papers, including books in the field of ultrafast optics and its applications. His current research interests are in monocycle photonics, attosecond optics, and their applications including biophotonics.

Dr. Yamashita is a member of the Japan Society of Applied Physics, the Physical Society of Japan, the Laser Society of Japan, the Biophysical Society of Japan, and the Optical Society of America. 